\title{
Serum Vitamin D Profile In Black African Men with Prostate Cancer at Tertiary Referral Facility in Sub-Saharan Africa
}

\author{
Andrew O. Odhiambo ${ }^{\mathrm{a}, \mathrm{b}}$, Gladwell W. Kiarie ${ }^{\mathrm{a}, \mathrm{b}}$, Mungai P Ngugi ${ }^{\mathrm{a}, \mathrm{b}}$, \\ Mark D. Joshi ${ }^{\mathrm{a}, \mathrm{b}}$ \\ ${ }^{a}$ Department of Clinical Medicine and Therapeutics, University of Nairobi (UoN) \\ ${ }^{b}$ Kenyatta National Hospital $(\mathrm{KNH})$
}

\begin{abstract}
:
Background: Considerable epidemiological, in vitro, in vivo and clinical data support an association between vitamin D deficiency and prostate cancer risk and outcome. Few studies have examined this association in African men with prostate cancer. The vitamin D status in patients with prostate cancer in Kenya is unknown. This study aimed to determine the profile of vitamin $D$ levels in patients with prostate cancer and to correlate this to patient and disease characteristics.

Methods: Hospital-based cross-sectional study that evaluated black African men with incident or 3-month prevalent histologically confirmed prostate cancer seeking ambulatory care at KNH. Medical history was obtained by direct interview and the information recorded in questionnaires. Treatment history, pre-diagnostic serum PSA and Gleason score were abstracted from patient records. Every participant had their anthropometric measurements taken and plasma samples drawn for 25-hydroxyvitamin D (25-VD) concentrations using the LIAISON@ 25-OH automated chemiluminescent immunoassay method. The relationship between age, body mass index, pre-diagnostic serum PSA and Gleason score on vitamin D status was evaluated using bivariate and multivariate analysis.

Results: 162 black African men were evaluated. The mean 25-VD was $19.15 \mathrm{ng} / \mathrm{ml}$ and 144 (88.9\%) men had vitamin $D$ deficiency $(25-\mathrm{VD}<30 \mathrm{ng} / \mathrm{ml}) .29(17.9 \%)$ were severely deficient $(25-\mathrm{VD}<10 \mathrm{ng} / \mathrm{ml}), 115(71 \%)$ were moderately deficient $(10-<30 \mathrm{ng} / \mathrm{ml})$ and $18(11.1 \%)$ were normal $(30-100 \mathrm{ng} / \mathrm{ml})$. Gleason scores $>7(\mathrm{OR}$ 2.9; 95\% CI 1.5-5.5, $p=0.001)$ and serum PSA $\geq 50 \mathrm{ng} / \mathrm{ml}(\mathrm{OR} 2.2 ; 95 \% \mathrm{CI} 1.7-5.1, p=0.014)$ were associated with vitamin D deficiency $(25-\mathrm{VD}<20 \mathrm{ng} / \mathrm{ml})$ whereas age and BMI were not. Adjusted for age, BMI and serum PSA levels, having Gleason scores > 7 was independently associated with vitamin D deficiency (OR 2.5; 95\% CI $1.2-4.9, p=0.01$ ).
\end{abstract}

Conclusion: Vitamin D deficiency is very common in black African men with prostate cancer, particularly in those with higher Gleason scores.

\section{Introduction}

Vitamin D is well known fundamental role in skeletal mineralization and calcium and phosphate homeostasis. In the recent years, vitamin D has been reported to have anticancer activity. Cell culture studies have demonstrated its protective role against cancer development. Normal and malignant prostate cells contain vitamin D receptors which mediates the antiproliferative action of vitamin D. In addition, vitamin D can cause apoptosis, induce differentiation, inhibit telomerase expression, inhibit tumor cell invasiveness and suppress tumor-induced angiogenesis. While $30-32 \mathrm{ng} / \mathrm{ml}$ are the common cut points for optimal vitamin D concentrations with respect to a variety of health outcomes, levels greater than 20ng/ are required to realize antitumor properties of vitamin $\mathrm{D}^{56}$

Epidemiological studies have suggested an inverse relationship between serum hydroxyvitamin D (25VD - a metabolite of vitamin D) and the risk of various cancers including prostate cancer. Garland et al, in a review article reported that vitamin D deficiency was associated with an increased risk of colon, breast and ovarian cancers. Various published articles have shown a consistent correlation between vitamin D deficiency and prostate cancer risk, aggressiveness and mortality

With regard to serum vitamin D profiles in prostate cancer, black African's have been under-studied with no peer- reviewed published articles. Majority of studies have been conducted in Caucasian and black American populations in who authors have reported a significant burden of vitamin D deficiency with seasonal variation being a major predictor of vitamin $\mathrm{D}$ deficiency.

It is assumed that countries in tropical Africa receive adequate sun-exposure but their darker skin pigment is known to impair endogenous vitamin D synthesis. Given that the vitamin D status in black African men with prostate cancer is not known, we sought to determine the prevalence of vitamin D deficiency in this sample and determine patient and disease characteristics that are associated with vitamin D deficiency. 


\subsection{Subjects}

\section{Materials And Methods}

We screened incident and 3-month prevalent cases with histologically confirmed prostate cancer seeking ambulatory care at a tertiary referral facility in Nairobi, Kenya. Excluded were those on current documented oral vitamin D supplementation; or those who lacked accompanying histology and pre-diagnostic serum prostate-specific antigen (PSA) records. Written, informed consent was sought.

Sample size was computed based on a prevalence study in a heterogeneous group of prostate cancer patient in USA.

\subsection{Data Collection}

Trained research assistants obtained socio-demographic data and medical history by direct interview followed by anthropometric measurements which were used to compute body mass index (BMI).Gleason scores and pre-diagnostic serum PSA levels were abstracted from patient records. A cut point of 30ng/ml was selected to define the lower limit of normal range of $25-\mathrm{VD}$ concentration in keeping with the accepted and validated normal laboratory reference ranges.

\subsection{Serum 25-VD assessment.}

Concentration of serum 25-VD was determined by determined by direct competitive chemiluminescent immunoassay (CMIA) using the LIAISON ${ }^{\circledR}$ platform in a private city laboratory with stringent internal and external quality control measure both locally and internationally.

\subsection{Statistical analyses}

Statistical analysis was done using Statistical Package for Social Sciences (SPSS) version 20.Continuous data was summarized into means, standard deviations, medians, whereas categorical data was summarized into proportions, frequencies and percentages. Data summaries were presented using tables, pie charts and graphs. Serum 25-VD was the dependent variable and was tested for association with age, BMI, Gleason score and serum pre-diagnostic PSA using Chi-Square test. Participants' likelihood of having serum 25VD deficiency $(<20 \mathrm{ng} / \mathrm{ml})$ was estimated using odds ratios. Variables were included as potential confounders in multivariate models if they were significantly associated with 25 -VD concentrations in unadjusted regression models. All the statistical tests were performed at $5 \%$ level of significance.

\subsection{Ethical considerations}

The study received institutional ethics and research committee approval prior to data collection.

\subsection{Funding source}

This study was funded privately and solely by the principal author. There are no conflicts of interest.

\section{Results}

A total of 192 cases of prostate cancer were screened for eligibility. One hundred and sixty three were enrolled and 29 excluded (18 diagnosed $>3$ months earlier than interview date, 7incomplete records, and 4 were on current oral vitamin D supplements).Out of those who met the inclusion criteria, one participants' blood sample was severely hemolysed and thus 162 subjects were available for analysis.

Age ranged from 47 to 96 year with a median age (IQR) of 68 (64-74) years. Majority of participants (48.8\%) were aged between 61 and 70years. Approximately a half (53.1\%) had a normal BMI. Majority (78.4\%) were receiving androgen deprivation therapy and $16 \%$ reported a positive family history of prostate cancer in a $1^{\text {st }}$ degree relative. The mean (SD) Gleason score was $7.15(1.27)$ with $43.2 \%$ of our sample having a score of greater than 7 . The median pre-diagnostic serum PSA was 63.9 with majority $(77.8 \%)$ having greater than 50 $\mathrm{ng} / \mathrm{ml}$. (Table 1).

Table 1: baseline descriptive characteristics

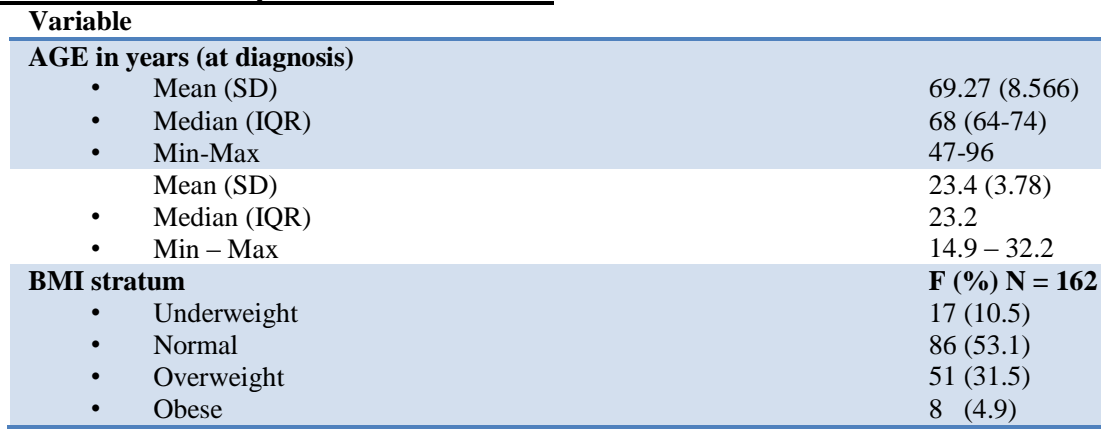




\begin{tabular}{|c|c|}
\hline \multicolumn{2}{|l|}{ Gleason score } \\
\hline - Mean (SD) & $7.15(1.27)$ \\
\hline - $\quad$ Median (IQR) & $7.00(6-8)$ \\
\hline - $\quad \operatorname{Min}-\operatorname{Max}$ & $4-10$ \\
\hline \multicolumn{2}{|l|}{ Gleason score stratum } \\
\hline - $\quad</=7$ & $92(56.8)$ \\
\hline - $\quad>7$ & $70(43.2)$ \\
\hline \multicolumn{2}{|l|}{ Serum PSA (pre-diagnostic) } \\
\hline - $\quad$ Mean $(\mathrm{SD})$ & $280.2(910)$ \\
\hline - $\quad$ Median (IQR) & $63.9(26-133)$ \\
\hline - $\quad \operatorname{Min}-$ Max & $0.07-8700$ \\
\hline Serum PSA stratum & $F(\%) N=162$ \\
\hline - $\leq 50$ & $36(24)$ \\
\hline - $\quad>50$ & $126(78)$ \\
\hline
\end{tabular}

Serum 25-VD concentrations ranged between 4.0 and $46.8 \mathrm{ng} / \mathrm{ml}$ with a mean (SD) of 19.15 (8.28). $11.1 \%$ had normal 25-VD concentrations while $71 \%$ had moderate deficiency and $17.9 \%$ had severe deficiency (Table 2).

Table 2: Vitamin D Profile

\begin{tabular}{|c|c|c|}
\hline Variable & & $95 \% \mathrm{CI}$ \\
\hline \multicolumn{3}{|l|}{ Serum 25-VD } \\
\hline - Mean (SD) & $19.15(8.28)$ & \\
\hline - $\quad$ Median (IQR) & $19.35(13.2-24.8)$ & \\
\hline - $\operatorname{Min}-\operatorname{Max}$ & $4-46.8$ & \\
\hline Serum 25-VD stratum & $F(\%) N=162$ & \\
\hline$\cdot \quad<10$ (severe deficiency) & $29(17.9)$ & $(12-23.8)$ \\
\hline - $\quad 10-<30$ (moderate deficiency) & $115(71)$ & $(64.01-77.99)$ \\
\hline - $\quad 30-100$ (Normal) & $18(11.1)$ & $(6.26-15.94)$ \\
\hline - $\quad>100$ (Toxic) & $0(0)$ & \\
\hline
\end{tabular}

In unadjusted bivariate analysis, participants with Gleason scores of greater than 7 where nearly thrice as likely to be vitamin D deficient. Participants with PSA levels of equal to or greater than 50 were twice as likely to be vitamin D deficient.

Table 3: Association between Serum 25-VD and Age, BMI, Gleason scores \& PSA

\begin{tabular}{|c|c|c|c|}
\hline \multicolumn{2}{|c|}{ VARIABLE } & Odds in 25-VD $<$ 20ng/ml (95\% CI) & P value \\
\hline \multirow{3}{*}{ Age Group } & $</=60$ yrs. & $\mathbf{1 . 0}$ & $\mathbf{0 . 3 0 3}$ \\
& $61-70$ yrs. & $\mathbf{0 . 6}(0.2-1.5)$ & $\mathbf{0 . 9 2 1}$ \\
& $71-80$ yrs. & $\mathbf{1 . 0 5}(0.4-2.9)$ & $\mathbf{0 . 2 9 7}$ \\
\hline \multirow{3}{*}{ BMI } & $>80$ yrs. & $\mathbf{2 . 1}(0.5-8.6)$ & $\mathbf{0 . 3 1 0}$ \\
& Under-wt. & $\mathbf{1 . 0}$ & $\mathbf{0 . 3 9 9}$ \\
& Normal & $\mathbf{0 . 6}(0.2-1.7)$ & $\mathbf{0 . 2 0 9}$ \\
\hline \multirow{2}{*}{ Gleason Score } & Over-wt. & $\mathbf{0 . 3}(0.05-1.9)$ & $\mathbf{0 . 0 0 1}$ \\
\hline \multirow{2}{*}{ PSA } & Obese & $\mathbf{1 . 0}$ & $\mathbf{2 . 9}(1.5-5.5)$ \\
\hline
\end{tabular}

Age and BMI had no effect on serum 25-VD concentrations. In a logistic regression model, the association between pre-diagnostic serum PSA and 25-VD deficiency was attenuated leaving Gleason score as the only independent predictor of vitamin D deficiency

\section{Discussion}

Based on databases of peer-reviewed published data, this is the first study of its kind in sub-Saharan Africa looking at the profile of serum vitamin D levels among black African men with prostate cancer. The study population was elderly, with a significant burden of advanced disease evidenced by the proportions with high Gleason Scores, high serum PSA receiving androgen deprivation therapy which is generally the palliative treatment modality of choice for advanced prostate cancer. The baseline characteristics of the study population were similar a descriptive series of prostate cancer patients at the same institution by Wasike et al who found a comparable mean age and similar proportions of those with advanced disease.

The mean serum vitamin D level in our study was low. Indeed, the prevalence of serum vitamin D deficiency is alarmingly high. Both Gleason Score and pre-diagnostic serum PSA were found to be significantly associated with vitamin D deficiency on bivariate analysis. However, Gleason score was the only independent 
predictor of vitamin D deficiency. Our study was not powered to show any effect of age and BMI on serum 25VD levels.

A similar cross-sectional study in New York, which evaluated a heterogeneous sample of prostate cancer patients found $72 \%$ and $62 \%$ prevalence of vitamin D deficiency in those with recurrent and localized disease respectively though they utilized a slightly higher cut-off. This was higher compared to our study sample whose mean 25-VD levels were $(19.15 \mathrm{ng} / \mathrm{ml})$ Vitamin D deficiency was more frequent in blacks and in those with recurrent disease ${ }^{10}$. It is probably evident from both studies that severe forms of disease are associated with vitamin D deficiency.

A study of similar design to ours evaluated those with non-metastatic prostate cancer disease found a lower prevalence of vitamin D deficiency. This is not surprising as vitamin D deficiency is more prevalent in those with advanced prostate cancer ${ }^{78}$. The finding of lower concentrations of vitamin D observed in our study is consistent with the known negative effect of skin pigmentation on endogenous synthesis of 25-VD. Other plausible explanations may include the fact that our study participants spent most time indoors, either seeking treatment and/or recuperating due to the impact of advanced disease and/or its treatments which has been observed in other patients with cancer receiving treatment.

Despite accepting results from different histopathology laboratories, Gleason scores are less likely to vary due to intricacies inculcated into the reporting criteria compared to serum PSA and hence a more reliable representative of aggressive disease in this study. This may explain why it was a stronger predictor than prediagnostic serum PSA in our study.

Our study did not find any association between age and BMI on serum 25-VD levels as it was not powered to do so. The aforementioned comparator studies ${ }^{10}{ }^{11}$ found similar findings with regard to age and BMI. Previous studies conducted in predominantly white populations with cancer have noted an inverse association between 25-VD concentration and BMI This association has been hypothesized to be related to the sun avoidance in those with higher BMI or sequestration of 25-VD in adipose tissue. However, other evidence exists to suggest that adiposity may not be a strong predictor of lower serum vitamin D levels in African Americans as compared to whites.

Indeed, our study had several strengths. As aforementioned, this is probably the first study evaluating serum vitamin D in black African prostate cancer patients in sub Saharan Africa. Secondly, the vitamin D assay technique that was employed is accurate, precise, well validated and comparable to the gold standard. The American association of Clinical Chemistry in July 2012 rated the performance of this method as excellent to world class. Comparator studies utilized a similar method.

Our study had some limitations. First, its major setback is the lack of local population reference ranges and a matched control group. This study is, therefore, unable to demonstrate whether vitamin D deficiency is more prevalent in those with prostate cancer than controls. Secondly, it had a relatively small sample size which was computed to meet the primary objective. Thus, the interpretation of data calls for caution and the exploratory analysis examining potential variables associated with vitamin D deficiency is limited. Thirdly, it did not address all potential confounders of that can affect serum 25-VD levels. For example, this study did not assess patterns of sun exposure, degree of skin pigmentation and the possibility of liver metastasis impairing hepatic synthesis of 25-VD. Lastly, pre-diagnostic serum PSA levels were not standardized as we accepted results from different laboratories. This may be responsible for the wide ranges and may have influenced our secondary explorations. Nevertheless, our study was able to determine the profile if serum vitamin D, prevalence of vitamin D deficiency and employ exploratory models to determine factors that might be associated with vitamin D deficiency.

In our sample of Black African men with prostate cancer, we found very low vitamin D levels and an alarmingly high prevalence of vitamin D deficiency higher than that observed in prostate cancer patients in the Northern latitudes. In addition, vitamin D deficiency was more common in those with aggressive disease.

We recommend further studies to explore the risk factors for vitamin D deficiency in prostate cancer patients and whether vitamin D replacement on may influence outcomes in those with vitamin D deficiency.

\section{Bibliography}

[1]. Ylikomi T, Laaksi I, Lou YR et al., Anti-proliferative action of vitamin D. Vitam Horm2002;64: 357-406.

[2]. Chen TC, Holick MF. Vitamin D and prostate cancer prevention and treatment. Trends Endocrinol Metab 2003;14: 423-30

[3]. Heany RP. Functional indices of vitamin D status and ramifications of vitamin D deficiency, AmJ ClinNutr 2004;66; 929-936

[4]. Bischoff-Ferrari HA, Giovanucci E, Willet WC et al. Estimation of optimal serum 25-hydroxyvitamin D for multiple health outcomes. AmJ ClinNutr 2006; 84: 18-28.

[5]. Garland C.F, Gorham E.D, Sharif B et al. Vitamin D for Cancer Prevention: Global Perspective, Ann Epidemiol2009;19: 468-483

[6]. Ahonen MH, Tenkanen L, Teppo L et al. Prostate Vitamin D and Prostate Cancer risk and prediagnostic serum 25-hydroxyvitamin D levels (Finland). Cancer Causes Control 2000;11: 847-52.

[7]. Li H, Stampfer MJ, Hollis JB, et al. A prospective study of plasma vitamin D metabolites, vitamin D receptor polymorphisms, and prostate cancer. PLOS:Medicine 2007;4 e103.

[8]. Corder EH, Guess HA, Hulka BS et al. Vitamin D and prostate cancer: a prediagnostic study with stored sera. Cancer Epidem, Biomar 1993;2: 467-472. 
[9]. Tretli S, Hernes E, Berg JP et al. Association between serum 25(OH)D and death from prostate cancer. BritJCancer 2009;10;100: 450-4.

[10]. Trump DL, Chadha MK, Sunga AY et al. Vitamin D deficiency and insufficiency among patients with prostate cancer. Department of Medicine, Roswell Park Cancer Institute, Buffalo, NY, USA. BJUI International 2009;104: 909-14.

[11]. Choo C.S, Mamedov A, Chung M et al. Vitamin D insufficiency is common in patients with nonmetastatic prostate cancer. Nutr Res2011;31: 21-6.

[12]. Tangpricha V, Natalia A, Colon BA et al. Prevalence of Vitamin D Deficiency in Patients Attending an Outpatient Cancer Care Clinic in Boston,Endocr Pract 2004;10: 292-293.

[13]. Ersfeld D.L, Rao D.S, Body J.J et al. Analytical and Clinical validation of 25-OH vitamin D assay for the LIAISON automated analyzer. ClinBiochem 2004;37: 867-74

[14]. ${ }^{1}$ Wasike and Magoha. Descriptive case series of patients presenting with cancer of the prostate and their management at Kenyatta National Hospital, Nairobi, Et Afr MedJSupplement 2007; 84(9)

[15]. Sunga A.Y, Trump D, Johnson L et al. Chemotherapy is linked to severe vitamin D deficiency in patients with colorectal cancer. J ClinOncol2010;31: 7255

[16]. Gupta D, Trukova P, Vashi G et al. Association of serum 25-hydroxy vitamin D and body mass index in cancer, J ClinOncol2009;27: 15s (supplement; abstract 6625)

[17]. Wortsman J, Matsouka LY, Chen TC et al. Decreased bioavailability of vitamin D in obesity, Am JClinNutr2000;72: 690-693

[18]. Compston J.E, Vedi S, Ledger J.E, et al. Vitamin D status and bone histomorphometry in gross obesity. Am JClinNutr 1981;34:2359-63

[19]. Epstein S, Bell NH, Shary J et al. Evidence that obesity does not influence the vitamin D-endocrine system in blacks. JBone MinerRes1986;1: 181-184.

[20]. Kiovula M.K, Turpeinen U, Laitinen P et al. Comparison of Automated 25-OH Vitamin D Immunoassays with Liquid Chromatography Isotope Dilution Tandem Mass Spectrometry, Clin Lab2012:58 